\title{
A New Technique for Conchal Cartilage Harvest
}

\author{
Joon Young Kim ${ }^{1}$, Ho Jik Yang ${ }^{1}$, Ji Won Jeong ${ }^{2}$ \\ ${ }^{1}$ Department of Plastic and Reconstructive Surgery, Eulji University Hospital, Daejeon; ${ }^{2}$ Doctorsmi Aesthetic Plastic Surgical Clinic, Daejeon, \\ Korea
}

The goal of auricular cartilage harvest is to obtain a sufficient amount for reconstruction and to minimize the change in ear shape. The cartilage can be harvested by a posterior or anterior approach, and each method has advantages and disadvantages. The posterior approach presents the advantage of scar concealment, but there are limits to the amount of cymba cartilage that may be harvested. In contrast, the anterior approach may cause a noticeable scar. However, as cartilage is collected, the anterior approach provides a view that facilitates the preservation ear structure. In addition, it is possible to obtain a greater amount of cartilage. From January 2014 to December 2015, we harvested auricular cartilage graft material in 17 patients. To prevent the development of trapdoor scars or linear scar contracture, short incisions were made on the superior border of the cymba and cavum. Two small and narrow incisions were made, resulting in suboptimal exposure of the surgical site, which heightens the potential for damaging the cartilage when using existing tools. To minimize this, the authors used a newly invented ball-type elevator. All patients recovered without complications after surgery and reported satisfaction with the shape of the ear.

Keywords Ear cartilage / Tissue and organ harvesting / Autograft

\author{
Correspondence: Ho Jik Yang \\ Department of Plastic and \\ Reconstructive Surgery, Eulji \\ University Hospital, 95 Dunsanseo-ro, \\ Seo-gu, Daejeon 35233, Korea \\ Tel: +82-42-611-3029 \\ Fax: +82-42-259-1111 \\ E-mail: drhjyang@eulji.ac.kr
}

No potential conflict of interest relevant to this article was reported.

Received: 18 Jun 2016 • Revised: 17 Dec 2016 • Accepted: 19 Dec 2016

pISSN: 2234-6163 • elSSN: 2234-6171 • https://doi.org/10.5999/aps.2017.44.2.166• Arch Plast Surg 2017;44:166-169

\section{INTRODUCTION}

Autologous cartilage has a high resistance to infection and a low rate of resorption, which makes it an ideal graft material for rhinoplasty [1]. Septal cartilage is generally considered the preferred graft material, being located at an optimal site. Its advantages include anatomic location within the same operative field and abundant availability of the cartilage [2]. However, the availability of the septal cartilage is limited in patients undergoing revision surgery, patients with a history of nasal trauma, or those with septal perforation. A congenitally limited distribution of septal cartilage may also affect availability [3]. In such cases, auricular cartilage is considered an appropriate alternative to septal cartilage. Typically, when auricular cartilage is utilized, conchal cartilage is preferred [4]. Several factors must be considered when harvesting conchal cartilage: harvesting an adequate amount, obtaining cartilage without inflicting damage during surgery, and ensuring that there are no postoperative changes to the shape of the ear [2].

To harvest conchal cartilage, either an anterior or posterior approach is used, with incision lines on the anterior or posterior surface of the ear, respectively. With a posterior approach, it is difficult to harvest an adequate amount of cartilage from the concha cymba. With an anterior approach, scar contracture or trapdoor scars can result because of the shape of the concave incision line [5]. The incision can be split into 2 parts, upper and lower, in order to circumvent the aforementioned disadvantages of the anterior approach. However, using 2 incision sites can re- 
sult in damage to the cartilage due to suboptimal surgical site access. Due to the concave shape of the conchal cartilage, pressure force occurs, and a sharp elevator may cause damage to the cartilage. Thus, we developed and utilized a ball-type elevator in order to avoid damaging the cartilage.

In this study, we employed an anterior approach in order to harvest a sufficient amount of conchal cartilage for rhinoplasty. Two incisions were made to minimize changes to the shape of the ear, and a ball-type elevator was used to minimize the damage to the cartilage during harvest. We achieved good results using this technique and hereby present our experience.

\section{IDEA}

From January 2014 to December 2015, auricular cartilage harvest operations were performed on 17 patients. The average patient age was 32 years (range, 19-51 years). Fourteen patients had a history of nasal surgery. Septoplasty was performed in 3 of the 14 patients with a surgical history. Secondary rhinoplasty was performed in 11 of the 14 patients, as they had undergone

\section{Fig. 1. Design of the incision line}

Incision lines were designed to mark the superior border of the concha cymba and concha cavum.

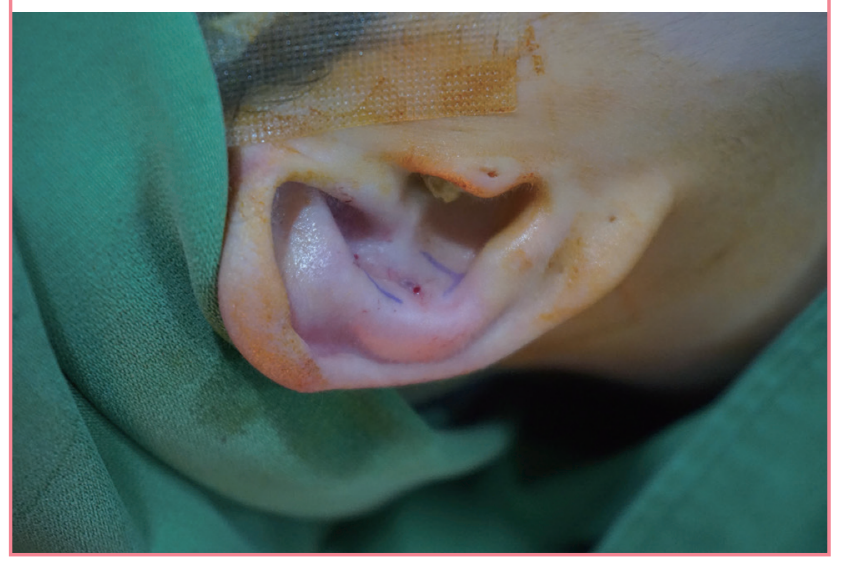

rhinoplasty with septoplasty in the past. Two patients had a history of nasal trauma and presented with a post-traumatic deformity. In these cases, the use of septal cartilage was deemed inappropriate. One patient had a secondary cleft lip and nose deformity, and conchal cartilage was used for alar wall reconstruction.

Surgery was performed under local anesthesia. The patient's face and both ears were exposed and prepared. For hemostasis and hydrodissection, 1\% lidocaine with 1:100,000 epinephrine was infiltrated into the subperichondrial plane above the conchal cartilage. Incision markings were made on the superior border of the concha cymba and concha cavum, with a length of approximately $1.5 \mathrm{~cm}$ (Fig. 1). The skin and soft tissue incision was made above the marks using a No. 15 blade. Further dissection was performed with dissecting scissors to expose the perichondrium. The perichondrial incision was made with a No. 15 blade and the subperichondrial plane was exposed. Prior hydrodissection facilitates this process. Initially, a pair of sharp dissecting scissors was used to dissect the anterior auricular flap of the conchal cartilage in the subperichondrial plane. Once the conchal cartilage was exposed, blunt dissection with a ball-type elevator (Fig. 2A) was performed and a wide skin flap including the perichondrium was elevated by a subperichondrial approach (Figs. 2, 3). The first upward incision was made on the cartilage using a No. 15 blade and then cut with the dissecting scissors. Blunt dissection with a ball-type elevator was performed to include the perichondrium on the posterior surface of the cartilage. The lower dissection was performed in similar fashion. The cartilage was harvested using a tissue forceps and dissecting scissors. Electrocautery was used for securing hemostasis and a 5-0 fast-absorbing suture was used for skin closure. Bolster dressing was applied with gauze and a silicon drain was maintained to prevent hematoma formation and skin necrosis. The drain was removed on the third postoperative day and sutures were removed on the seventh day.

An adequate amount of cartilage was harvested from 1 ear in 16 patients. Only 1 patient required cartilage harvest from both

\section{Fig. 2 Intraoperative photograph}

(A) A ball-type elevator was used for blunt dissection of the subperichondrial plane. (B) The flap was elevated after blunt dissection of the subperichondrial plane using a ball-type elevator. (C) Harvested conchal cartilage.
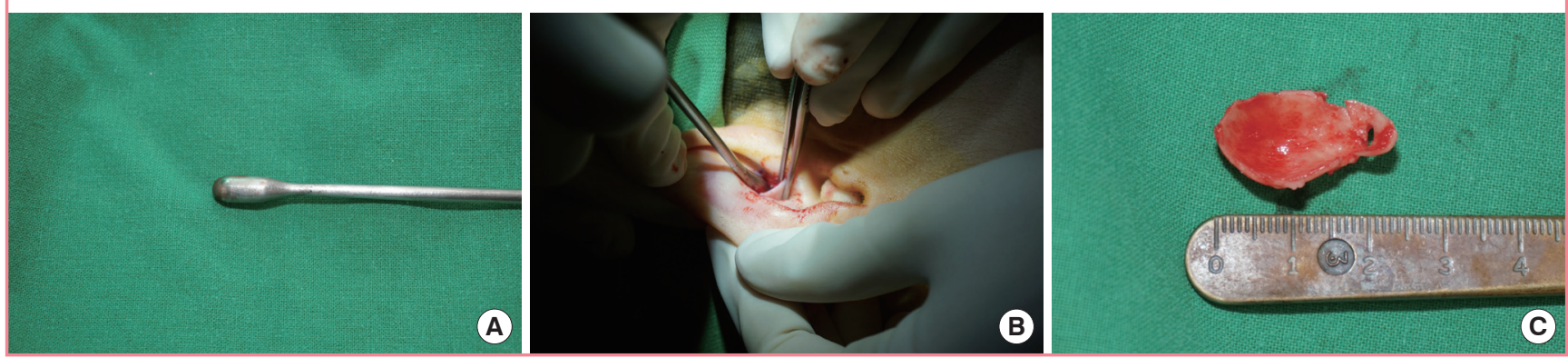


\section{Fig. 3. Schematic drawing of the operation}

The illustration depicts the incision lines (red lines) for the auricular cartilage harvest (blue dotted line).

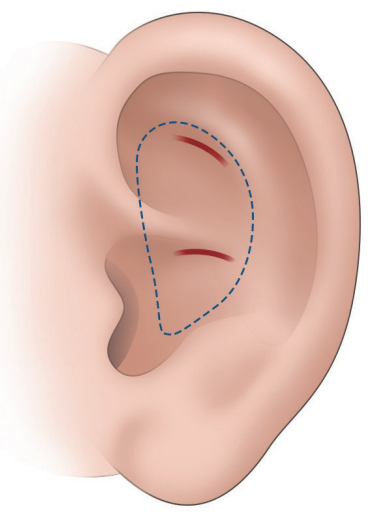

ears. The average dimensions of the harvested cartilage were $22.8 \mathrm{~mm}$ in height and $13.6 \mathrm{~mm}$ in width. Postoperative hematoma, skin necrosis, infection, or other complications were not observed in any of the patients. The average follow-up period was 10 months, with a range of 3 to 24 months. There were no reports of hypertrophic scar or keloid formation during the follow-up period. All 17 patients were satisfied with the appearance of the donor site after surgery (Fig. 4). In only 1 case was there damage to the cartilage.

\section{DISCUSSION}

The ideal grafting material should be biocompatible, highly resistant to infection, non-resorbable, easily manipulated, structurally sound, non-carcinogenic, easy to obtain, cost-effective, and similar to the native framework. Graft material comes in 3 types: autografts (native cartilage, bone, fascia, or dermis), homografts (irradiated rib or pooled acellular dermis), and alloplasts (Silastic, Mersilene, Gore-Tex, or Medpor) [6]. Homografts may have problems with resorption and warping [7]. Alloplasts may have an increased incidence of infection and extrusion [8]. The use of autografts presents several advantages: they are easily manipulated and highly resistant to infection, and have a low rate of resorption [1]. Autologous cartilage graft material can be harvested from various areas, and these typically include septal cartilage, auricular cartilage, and costal cartilage [9].

Septal cartilage is the primary choice of graft material in rhinoplasty. It can be used in all areas of rhinoplasty, including reconstruction of the nasal tip, dorsal onlay, columellar strut, and nasal spreader grafts. Septal cartilage has several advantages. It is easy to harvest, it is associated with low morbidity, and it is

\section{Fig. 4. A patient before and after cartilage harvest}

A 25-year-old woman who underwent conchal cartilage harvest. (A) Lateral view of the donor ear preoperatively. (B) Lateral view of the donor ear 1 year postoperatively.
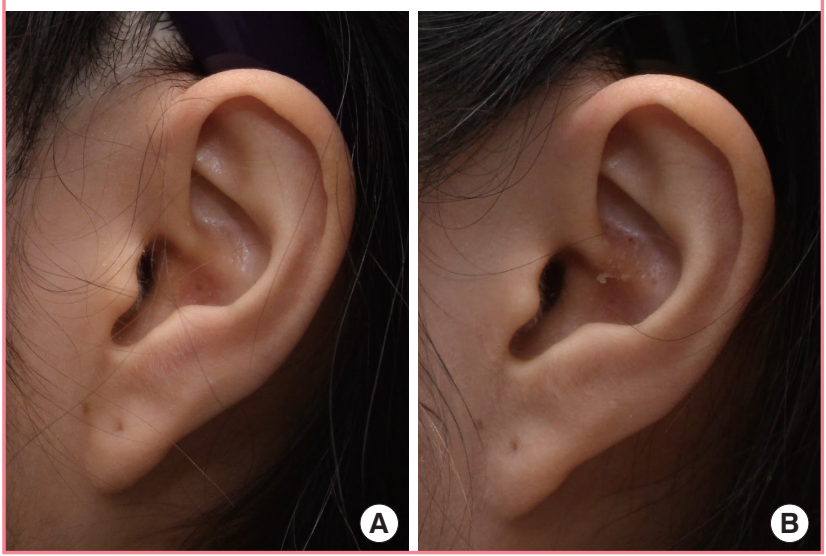

drawn from the same operative field [10]. However, the use of septal cartilage is limited in patients undergoing secondary revision surgery, patients with a history of trauma to the area, and those with nasal septum perforation [3].

Costal cartilage is utilized when a large amount of graft material or strong structural support is required [11]. However, the use of costal cartilage as graft material requires an additional operative field, and is associated with an increased likelihood of chest wall infection, hematoma, anatomic deformity, and pneumothorax [12].

Auricular cartilage is generally utilized alone or as a supplemental source of graft material when the availability of the primary graft material is limited [2]. Auricular cartilage is often used as a graft material for East Asian patients, whose noses may contain limited septal cartilage, or in patients where septal cartilage is limited for other reasons [13]. Auricular cartilage is used for the reconstruction of the nasal tip, dorsal onlay, alar contour, and lower lateral cartilages [14]. Auricular cartilage presents the advantage of being harvested from the same operative field, with low morbidity of the donor site. Moreover, warping is uncommon and the curved three-dimensional structure of the auricular cartilage is similar to the lateral crura. Conchal cartilage is the most common type of auricular cartilage that is used as a graft material. The concha is divided into the superior cymba and the inferior cavum. Cavum cartilage is typically used for nasal tip plasty. Cymba cartilage is more curved, more delicate, and narrower than cavum cartilage [13].

Although the posterior approach for auricular cartilage harvest can provide better cosmetic results by hiding the scar more efficiently, only a limited amount of cartilage can be harvested. The anterior approach allows easier dissection, with better visible 
landmarks for preserving the shape of the ear [2], and a larger amount of cymba cartilage can be harvested. Furthermore, the anterior approach allows the preservation of the neurovascular system distributed along the posterior surface of the ear. One of the disadvantages of this approach is the visibility of a scar on the anterior surface of the ear. To minimize this, an incision is made along the conchal rim to hide the scar within the convolution of the antihelix [15]. Regardless of the effort, anterior scars are easily observed in East Asian patients, and deformation in the shape of the ear is more likely [13]. Other problems include trapdoor scar formation due to the concave incision line and linear scar contracture secondary to the linear incision line. To prevent the development of linear scar contracture, the incision line can be divided into 2 short incision lines on the superior border of the conchal cymba and cavum. Of course, the incision length is dependent on the size of the ear.

The novel technique presented here uses 2 short incisions (1 above and 1 below) to harvest the cartilage, with limited exposure of the middle portion of the cartilage. Thus, cartilage damage during harvest is more likely. In addition, existing sharp tools such as the Freer elevator may act as a knife when the pressure vector is inconsistent with the shape of the cartilage. This results in damage to the cartilage. Therefore, less utility can be derived from the cartilage after harvest. A blunt dissection with a balltype elevator can be used to minimize damage to the cartilage. Two short incisions in addition to the incision of the cartilage also facilitate easier dissection. This helps to harvest the cartilage without damage. By preserving the perichondrium on the anterior side of the ear, deformation of the ear can be avoided.

In this study, we used a novel technique with 2 short incisions for conchal cartilage harvest. We minimized damage to the cartilage during harvest by using a ball-type elevator and limited postoperative changes to the shape of the ear shape by employing a rather simple surgical procedure.

\section{REFERENCES}

1. Endo T, Nakayama Y, Ito Y. Augmentation rhinoplasty: observations on 1200 cases. Plast Reconstr Surg 1991;87:54-9.

2. Lee M, Callahan S, Cochran CS. Auricular cartilage: harvest technique and versatility in rhinoplasty. Am J Otolaryngol 2011;32:547-52.

3. Cochran CS, DeFatta RJ. Tragal cartilage grafts in rhinoplasty: a viable alternative in the graft-depleted patient. Otolaryngol Head Neck Surg 2008;138:166-9.

4. Boccieri A, Marano A. The conchal cartilage graft in nasal reconstruction. J Plast Reconstr Aesthet Surg 2007;60:18894.

5. Grobbelaar AO, Matti BA, Nicolle FV. Donor site morbidity post-conchal cartilage grafting. Aesthetic Plast Surg 1997; 21:90-2.

6. Steiger JD, Bloom J, Becker DG. Auricular cartilage grafts. Oper Tech Otolayngol Head Neck Surg 2008; 19:267-72.

7. Adams WP Jr, Rohrich RJ, Gunter JP, et al. The rate of warping in irradiated and nonirradiated homograft rib cartilage: a controlled comparison and clinical implications. Plast Reconstr Surg 1999;103:265-70.

8. Romo T 3rd, Kwak ES, Sclafani AP. Revision rhinoplasty using porous high-density polyethylene implants to reestablish ethnic identity. Aesthetic Plast Surg 2006;30:679-84.

9. Gunter JP, Landecker A, Cochran CS. Frequently used grafts in rhinoplasty: nomenclature and analysis. Plast Reconstr Surg 2006;118:14e-29e.

10. Sajjadian A, Rubinstein R, Naghshineh N. Current status of grafts and implants in rhinoplasty: part I. Autologous grafts. Plast Reconstr Surg 2010;125:40e-49e.

11. Moshaver A, Gantous A. The use of autogenous costal cartilage graft in septorhinoplasty. Otolaryngol Head Neck Surg 2007;137:862-7.

12. Thomson HG, Kim TY, Ein SH. Residual problems in chest donor sites after microtia reconstruction: a long-term study. Plast Reconstr Surg 1995;95:961-8.

13. Han K, Kim J, Son D, et al. How to harvest the maximal amount of conchal cartilage grafts. J Plast Reconstr Aesthet Surg 2008;61:1465-71.

14. Rohrich RJ, Ahmad J. Plastic surgery. In: Neligan P, editor. Plastic surgery. London: Elsevier Saunders; 2013. p.395-6.

15. Erol OO. New modification in otoplasty: anterior approach. Plast Reconstr Surg 2001;107:193-202. 